\title{
Morfometría geométrica en cinco especies de Buthidae y Scorpionidae (Arachnida: Scorpiones) de Venezuela
}

\section{Geometric morphometrics in five species of Buthidae and Scorpionidae (Arachnida: Scorpiones) from Venezuela}

\author{
Walter Y. Bechara y Jonathan Liria ${ }^{凶}$ \\ Departamento de Biología, Facultad Experimental de Ciencias y Tecnología, Universidad de Carabobo. Valencia, Carabobo 2005 Venezuela. \\ 囚jonathan.liria@gmail.com
}

\begin{abstract}
Resumen. En Venezuela, la escorpiofauna consta de 17 géneros y 202 especies. En algunos grupos la taxonomía no ha sido aclarada; por lo tanto, son necesarias investigaciones adicionales que contribuyan a mejorar el conocimiento sobre la identidad taxonómica y relaciones entre sus componentes. La morfometría geométrica es una herramienta que ha sido utilizada como apoyo a la sistemática en distintos organismos. El objetivo del presente trabajo fue analizar la conformación y tamaño isométrico en 5 especies de escorpiones. Se fotografiaron 266 individuos de 4 especies de Buthidae: Centruroides testaceus, Rhopalurus laticauda, Tityus clathratus y T. discrepans, y una de Scorpionidae: Tarsoporosus yustizi. Se registraron configuraciones de coordenadas $(x, y)$ en 5 estructuras a partir de puntos anatómicos de referencia (PAR) y PAR deslizantes. Los resultados mostraron separación completa de las especies de Buthidae por medio de tricobotrias dorsales en la patela del pedipalpo, y una consistente separación en el nivel familiar en las estructuras restantes. Las placas delgadas permitieron la visualización de compresión horizontal del esternón en las especies de Buthidae y compresión vertical en la de Scorpionidae. Se determinó la similitud de coxas, quela y caparazón entre $R$. laticauda y $C$. testaceus. La disposición de tricobotrias y la conformación del esternón permitió la diferenciación entre $T$. discrepans y T. clathratus.
\end{abstract}

Palabras clave: Tityus, Rhopalurus, Centruroides, Tarsoporosus, puntos anatómicos, procrustes, patela, pedipalpo, esternón.

\begin{abstract}
The Venezuelan scorpionfauna comprises 17 genera and 202 species. In some taxa the taxonomy is unclear, and additional studies that contribute to the knowledge about taxonomic identity and relationships are necessary. Geometric morphometrics is a tool that has been used as support to the systematics in different organisms. The goal was to analize the isometric size and conformation in five scorpions species. We photographed 266 individuals of four speceis of Buthidae: Centruroides testaceus, Rhopalurus laticauda, Tityus clathratus and T. discrepans, and one species of Scorpionidae: Tarsoporosus yustizi. Coordinate $(x, y)$ configurations from landmarks and semilandmarks were registered in five structures. The results showed the complete separation in Buthidae species through dorsal trichobotria on patella pedipalp; and a consistent separation at family level in the remaining structures. The thin plate spline showed the sternum horizontal compression in the Buthidae species and the vertical compression in Scorpionidae. Similarity in coxae, chelae and caparace between $R$. laticauda and $C$. testaceus was determined. The trichobotria disposition and the sternum conformation allows to discriminate $T$. discrepans and $T$. clathratus.
\end{abstract}

Key words: Tityus, Rhopalurus, Centruroides, Tarsoporosus, landmarks, procrustes, patella, pedipalp, sternum.

\section{Introducción}

Los arácnidos constituyen la clase más diversa de quelicerados (aprox. 74000 especies), dentro de los cuales el orden Scorpiones cuenta con más de 1500 especies. Los escorpiones son un pequeño grupo de artrópodos terrestres que tienen hábitos sigilosos y nocturnos; durante el día se ocultan bajo piedras, troncos y grietas o en galerías

Recibido: 22 agosto 2011; 28 octubre 2011 que cavan en el sustrato. Tienen una actividad estacional marcada; la mayoría de las especies es estival. La mayor parte del tiempo se encuentran en sus refugios de donde sólo salen para alimentarse y reproducirse (Polis, 1990; Warburg y Polis, 1990). En general, los escorpiones son estenoicos y poco móviles (Lourenço, 1994, 2002; Prendini, 2001).

El orden Scorpiones se considera tradicionalmente conformado por 9 familias; sin embargo, revisiones recientes sobre la taxonomía de categorías superiores revelan que podrían ser reconocidas entre 12 y 20 familias (Stockwell, 
1992; Lourenço y Otero, 1998; Lourenço, 2000; Fet et al., 2000; Prendini y Wheeler, 2005). En Venezuela, la escorpiofauna comprende 17 géneros y 202 especies conocidas (Rojas-Runjaic y Becerra, 2008). En algunas especies se requieren investigaciones adicionales que contribuyan a mejorar el conocimiento sobre la identidad taxonómica, distribución geográfica y las relaciones de algunos de sus componentes. Las especies venezolanas se clasifican en 5 familias: Buthidae, Chactidae, Euscorpiidae, Hemiscorpiidae y Scorpionidae. Rojas-Runjaic y Becerra (2008) indican que en el país las Scorpionidae están representadas por el género Tarsoporosus (4 spp.), mientras que las Buthidae incluyen 7 géneros Ananteris (31 spp.), Centruroides (2 spp.), Isometrus (1 sp.), Mesotityus (1 sp.), Microtityus (8 spp.), Rhopalurus (2 spp.) y Tityus (58 spp.). Este último es el de mayor importancia médica, particularmente en el norte del país, pues contiene especies cuyo veneno es altamente tóxico (De Sousa et al., 1996, 2000; Machado y Rodríguez-Acosta, 1997; Reyes-Lugo y RodríguezAcosta, 2001; Guinand et al., 2004). A pesar del innegable interés científico y social, muchos de los miembros de este género no han sido debidamente estudiados desde el punto de vista taxonómico (Rojas-Runjaic y De Armas, 2007).

Una herramienta que podría contribuir a los estudios taxonómicos de Scorpiones es la morfometría geométrica, la cual está definida como la fusión entre la geometría y la biología, comprendiendo el estudio de la forma en un espacio bi o tridimensional (Bookstein, 1982). Recientemente, se ha usado en diversos organismos vegetales y animales, y particularmente en artrópodos con importancia médica, como triatominos (Triatominae), flebótomos (Phlebotominae) y mosquitos (Culicidae). Ha sido empleada en estudios de estructura poblacional (De la Riva et al., 2001; Belen et al., 2004; Dvorak et al., 2006), para identificación de especies (Matías et al., 2001; Villegas et al., 2002; Calle et al., 2008), o detectar diferencias entre parentales de campo y descendientes de laboratorio (Jaramillo y Wolff, 2002), y en estudios filogenéticos (Soto-Vivas et al., 2011).

Por lo anteriormente expuesto, se propone realizar un estudio morfogeométrico en 5 especies de Buthidae y Scorpionidae, con el fin de describir cuantitativamente la conformación y tamaño de estructuras como quela, patela, caparazón, esternón y coxas, contribuyendo al componente taxonómico y futuras investigaciones de variabilidad intraespecífica en el orden Scorpiones.

\section{Materiales y métodos}

Adquisición de datos. Se revisó material depositado en el Museo de Biología de la Universidad de Carabobo y en la Colección del Laboratorio de Neurofarmacología Celular del Instituto Venezolano de Investigaciones Científicas.
Se acondicionó un laboratorio en completa oscuridad y los ejemplares fueron iluminados con 2 tubos de luz ultravioleta de 6 watt, marca Xilco ${ }^{\circledR}$. Este tipo de iluminación permitió detallar características en la morfoescultura de la superficie de los ejemplares, quetotaxia y tricobotriotaxia (Prendini, 2003a, 2003b, 2003c; Volschenk, 2005). Seguidamente se fotografiaron digitalmente la quela y patela del pedipalpo, caparazón, esternón y coxas de 266 ejemplares en 4 especies de Buthidae: Tityus discrepans Karsch, 1879 $(\mathrm{n}=64), T$. clathratus Koch, $1845(\mathrm{n}=31)$, Rhopalurus laticauda Thorell, $1876(\mathrm{n}=45)$ y Centruroides testaceus (DeGeer, 1778) $(\mathrm{n}=96)$, y una especie de Scorpionidae: Tarsoporosus yustizi (González-Sponga, 1983) ( $\mathrm{n}=30)$. En cada estructura se ubicaron puntos anatómicos de referencia (PAR), los cuales corresponden con el tipo I sensu Bookstein (1991) en tricobotrias de patela $\left(d_{1-5}, i\right)$ y coxas (II, III y IV) y tipo II-III en quela del pedipalpo, esternón y prosoma (Fig. 1). Para cada análisis, el número de ejemplares varió dependiendo de la condición de algunas estructuras y en el caso de T. yustizi, éste no fue incluido en el estudio de patela, puesto que la disposición y el número de tricobotrias fue distinto respecto a Buthidae. En todos los análisis se mantuvo la premisa de contar con más ejemplares que el doble de PAR por estructura. La configuración de los pares de coordenadas $x, y$ se capturó mediante la ayuda del programa tpsDig (Rohlf, 2007). En el caso de quela, esternón y caparazón, se utilizó el programa MakeFan (Sheets, 2005c) para producir radios equidistantes, que sirvieron como líneas guía para digitalizar el contorno. Análisis de morfometría geométrica. A partir de las matrices por estructura de 266 configuraciones de coordenadas geométricas de PAR, se realizó el análisis generalizado de procrustes (AGP) con el programa CoordGen (Sheets, 2005a) y se extrajeron las variables de conformación (partial warps $=\mathrm{Pw})$ y el tamaño centroide $(\mathrm{CS})$. En el caso de los contornos, se utilizó TPSRelw (Rolhf, 2003) para realizar el AGP con PAR deslizantes (Bookstein, 1996). Las matrices de Pw se usaron para análisis de variables canónicas (AVC) con el programa CVAGen (Sheets, 2005b), mediante el cual también se obtuvo la proporción de grupos (= especies) reclasificados y MANOVA por cada eje canónico (Zelditch et al., 2004; van der Molen et al., 2007). Las diferencias en los valores de CS entre las especies fueron analizadas mediante la prueba de Kruskall-Wallis $(p<0.05)$.

\section{Resultados}

Patela del pedipalpo. Se encontraron diferencias significativas $(\mathrm{P}<0.001)$ entre el tamaño isométrico de todas las especies con excepción de $C$. testaceus y $R$. laticauda; siendo T. clathratus $(3.06 \mathrm{~mm})$ la de menor tamaño, seguida 

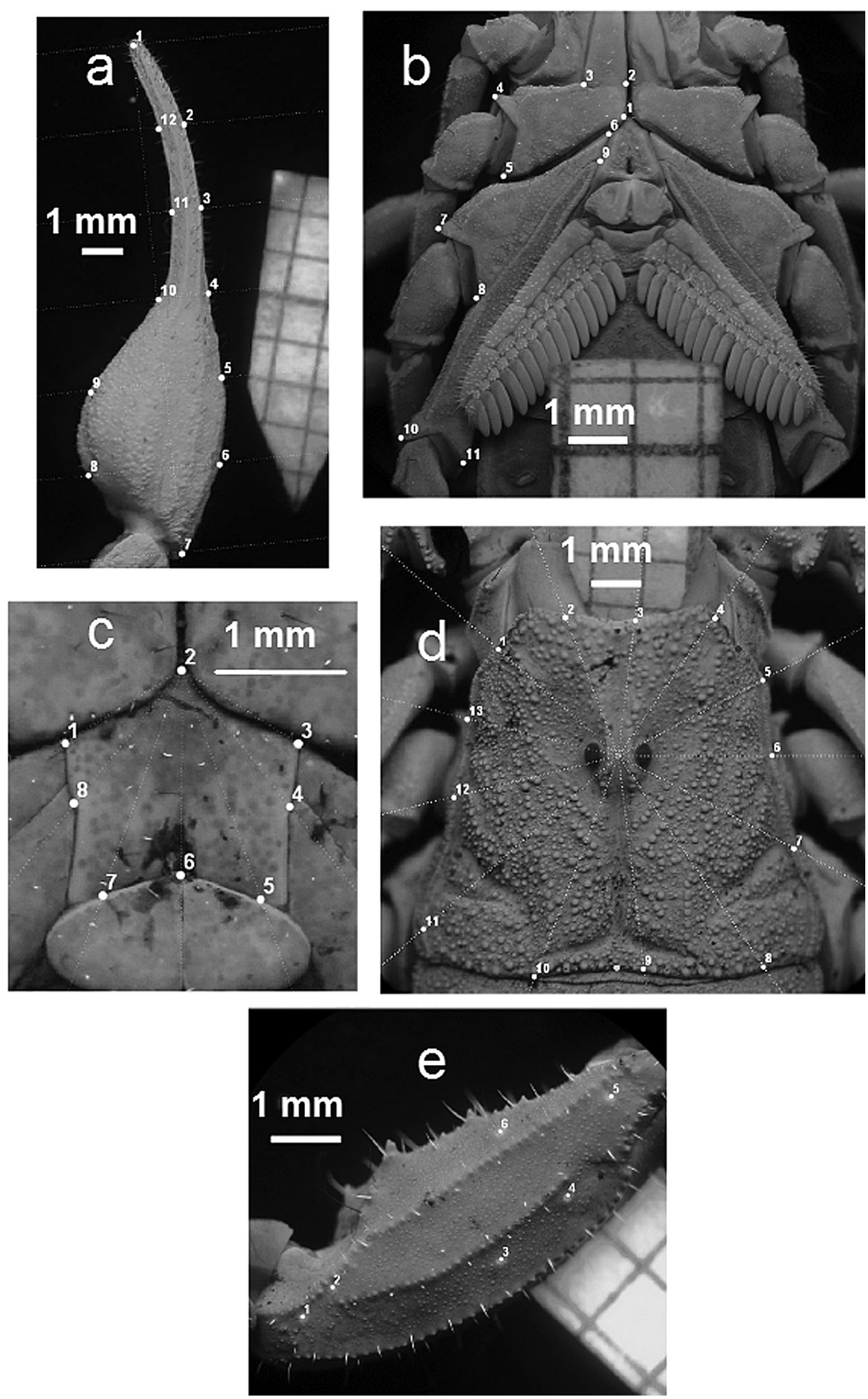

Figura 1. Microfotografías mostrando la disposición de los PAR en: a, quela de R. laticauda (PAR 1-12); b, coxas II-IV de T. clathratus (PAR 1-12); c, esternón de T. yustizi (PAR 1-8); d, caparazón de R. laticauda (PAR 1-13), y e, patela del pedipalpo de T. discrepans (PAR 1-6). 
de C. testaceus $(3.97 \mathrm{~mm})$, R. laticauda $(3.98 \mathrm{~mm})$ y T. discrepans $(4.85 \mathrm{~mm})$. La clasificación de los 233 individuos en los grupos a priori se basó según la identificación de las especies por la morfología tradicional y la reclasificación $a$ posteriori depende de las distancias de Mahalanobis entre cada individuo y la media de cada especie. Se presentan los resultados de la prueba de reclasificación en cada eje discriminante del AVC: Eje 1 Wilks lambda $(\Lambda)$ 0.0295, $\chi^{2}$ 619.82, 24 grados de libertad y $p<0.0001, \Lambda^{2}=0.3393$, $\chi 2190.23,14 \mathrm{gl} p<0.0001$, y $\Lambda^{3}=0.7945, \chi^{2} 40.47,6 \mathrm{gl}$ y $p<0.0001$. Los individuos reclasificados fueron casi perfectamente asignados al 97\% en T. clathratus (29/30) y $T$. discrepans (61/63), seguidos de $95 \%$ en $R$. laticauda (43/45) y $92 \%$ C. testaceus (88/95). El diagrama factorial del AVC (Fig. 2) muestra la separación de T. discrepans y C. testaceus, con solapamiento parcial entre T. clathratus y R. laticauda; la función de interpolación de placas delgadas (thin plate spline, TPS) permitió inferir las diferencias en conformación como deformaciones de los 6 puntos en una rejilla calculada con respecto a la configuración consenso (CC). En esta se aprecia la similitud entre la configuración de $T$. clathratus con respecto a $\mathrm{CC}$, a éste le sigue $C$. testaceus donde se presenta poca variación, con solo un leve desplazamiento de los PAR 3 (tricobotria $d_{3}$ ), $4\left(d_{4}\right)$ y 6 (tricobrotria interna $i$ ); en $R$. laticauda el PAR 4 se mueve en el sentido diagonal hacia la derecha, el PAR 6, a su vez, lo hace en dirección vertical, mientras que el PAR $5\left(d_{5}\right)$ se acerca de manera horizontal al 4 y 6 ; finalmente, $T$. discrepans muestra la configuración más alejada de la CC, donde los PAR $1\left(d_{1}\right)$ y $2\left(d_{2}\right)$ se acercan entre sí, desplazándose horizontalmente; luego, el PAR 4 se desplaza en sentido diagonal hacia la izquierda, y finalmente el PAR 5 se mueve horizontalmente hacia la derecha, alejándose del PAR 4.

Coxas. Se encontraron diferencias significativas $(p<0.05)$ entre el tamaño isométrico de todas las especies con excepción de T. clathratus y T. yustizi; siendo los de menor tamaño T. yustizi $(7.20 \mathrm{~mm})$ y $T$. clathratus $(7.62 \mathrm{~mm})$; seguidamente $C$. testaceus $(9.10 \mathrm{~mm})$ y $R$. laticauda $(9.83$ $\mathrm{mm})$, y finalmente $T$. discrepans $(11.71 \mathrm{~mm})$ con el mayor tamaño. Se presentan los resultados de la prueba de reclasificación en cada eje discriminante del AVC: $\Lambda^{1}=0.0004$, $\chi^{2} 1967.91,72 \mathrm{gl} p<0.0001, \Lambda^{2}=0.029, \chi^{2} 890.61,51 \mathrm{gl} p$ $<0.0001, \Lambda^{3}=0.2116, \chi 2390.57,32 \mathrm{gl}$ y $p<0.0001$ y $\Lambda^{4}=$ $0.5541, \chi^{2} 148.50,15 \mathrm{gl}$ y $p<0.0001$. La clasificación de 264 individuos fue perfecta al 100\% en T. yustizi (29/29), seguida de $98 \%$ C. testaceus $(93 / 95)$ y $R$. laticauda (44/45), 95\% en $T$. discrepans (61/64) y 94\% T. clathratus (29/31). El AVC (Fig. 3) muestra la separación de T. yustizi, de 2 grupos: uno conformado por $R$. laticuda y $C$. testaceus y otro por T. discrepans y T. clathratus. Los PAR 3 y 6 en C. testaceus muestran desplazamientos hacia los PAR 2 y 1 respectivamente, luego el PAR 9 se desplaza hacia el 8.
Estos desplazamientos ocurren en $R$. laticauda, pero adicionalmente el PAR 2 se acerca al 3. Luego en T. clathratus el PAR 3 se desplaza hacia el 5, mientras que el 1 se dirige al 3. Por otra parte en T. discrepans, el PAR 3 se mueve hacia 4 y 5 , el PAR 1 hacia el 2, y el PAR 6 hacia el 9. Finalmente, en T. yustizi se aprecia el PAR 1 alejándose del 3, y el PAR 2 del 5, luego el PAR 6 presenta la mayor deformación, desplazándose en dirección contraria al PAR 1 y acercándose al 7, este último se aleja del 8, el PAR 9 se mueve horizontalmente alejándose del 5 y por último el 10 se mueve hacia el 7.

Esternón. Se encontraron diferencias significativas $(p<$ $0.05)$ entre el tamaño isométrico de todas las especies; $T$. clathratus $(1.29 \mathrm{~mm})$ es la de menor tamaño, seguida por C. testaceus $(1.49 \mathrm{~mm})$, luego $R$. laticauda $(1.88 \mathrm{~mm}), T$. discrepans $(2.14 \mathrm{~mm})$, y finalmente T. yustizi $(2.54 \mathrm{~mm})$. Se presentan los resultados de la prueba de reclasificación en cada eje discriminante del AVC: $\Lambda^{1}=0.0149, \chi^{2} 1070.08$, $48 \mathrm{gl} p<0.0001, \Lambda^{2}=0.3102, \chi 2297.88,33 \mathrm{gl} p<0.0001$, $\Lambda^{3}=0.6665, \chi 2103.26,20 \mathrm{gl} \mathrm{y} p<0.0001$ у $\Lambda^{4}=0.8788$, $\chi 232.89,9 \mathrm{gl}$ y $p<0.001$. La clasificación de 264 individuos fue perfecta al 100\% en T. yustizi (29/29), seguida de $90 \%$ en T. clathratus (28/31), $67 \%$ en R. laticauda (30/45) y $T$. discrepans (43/64) y finalmente $65 \%$ en C. testaceus (62/95). El AVC (Fig. 4) muestra la separación de T. yustizi y T. clathratus y solapamiento entre las demás especies. En T. yustizi presenta la mayor variación respecto a los demás taxa: los PAR 1, 3, 4 y 8, se alejan del 2 aproximándose a 5 y 7 , los cuales a su vez se desplazan acercándose a 4 y 8 ; por último los PAR 2 y 6 se acercan entre si. En T. discrepans no se aprecia deformación de la rejilla, luego en $T$. clathratus se muestra una leve variación, específicamente en el PAR 2, con desplazamiento en el eje vertical alejándose de los PAR 1 y 3 ; seguidamente el 5 y 7 se acercan al 6 . Por otro lado, las configuraciones de $C$. testaceus y $R$. laticauda comparten cierto grado de similitud, presentándose en ambas el mismo patrón de desplazamiento de los PAR 1, 3, 4 y 8, que se desplazan hacia el 2. Luego los PAR 5 y 7 se acercan entre sí, y se alejan de 4 y 8 respectivamente.

Caparazón. Se encontraron diferencias significativas $(p<$ 0.05 ) entre el tamaño isométrico de todas las especies, con el menor tamaño para T. clathratus $(7.94 \mathrm{~mm})$, seguido de T. yustizi $(9.72 \mathrm{~mm})$, luego C. testaceus $(9.81 \mathrm{~mm})$, R. laticauda $(11.01 \mathrm{~mm})$ y por último T. discrepans $(12.19 \mathrm{~mm})$. Se presentan los resultados de la prueba de reclasificación en cada eje discriminante del AVC: $\Lambda^{1}=0.0144, \chi 21062.95$, $88 \mathrm{gl} p<0.0001, \Lambda^{2}=0.1116, \chi 2547.18,63 \mathrm{gl} p<0.0001$, $\Lambda^{3}=0.3960, \chi^{2} 231.15,40 \mathrm{gl}$ y $p<0.0001$ y $\Lambda^{4}=0.8010, \chi^{2}$ $55.40,19 \mathrm{gl}$ y $p<0.0001$. La clasificación de 264 individuos fue casi perfecta con $97 \%$ en T. yustizi (28/29), seguida de $84 \%$ T. discrepans (54/64), 83\% T. clathratus (26/31), 81\% R. laticauda (36/45) y $80 \%$ en C. testaceus (77/95). El AVC 

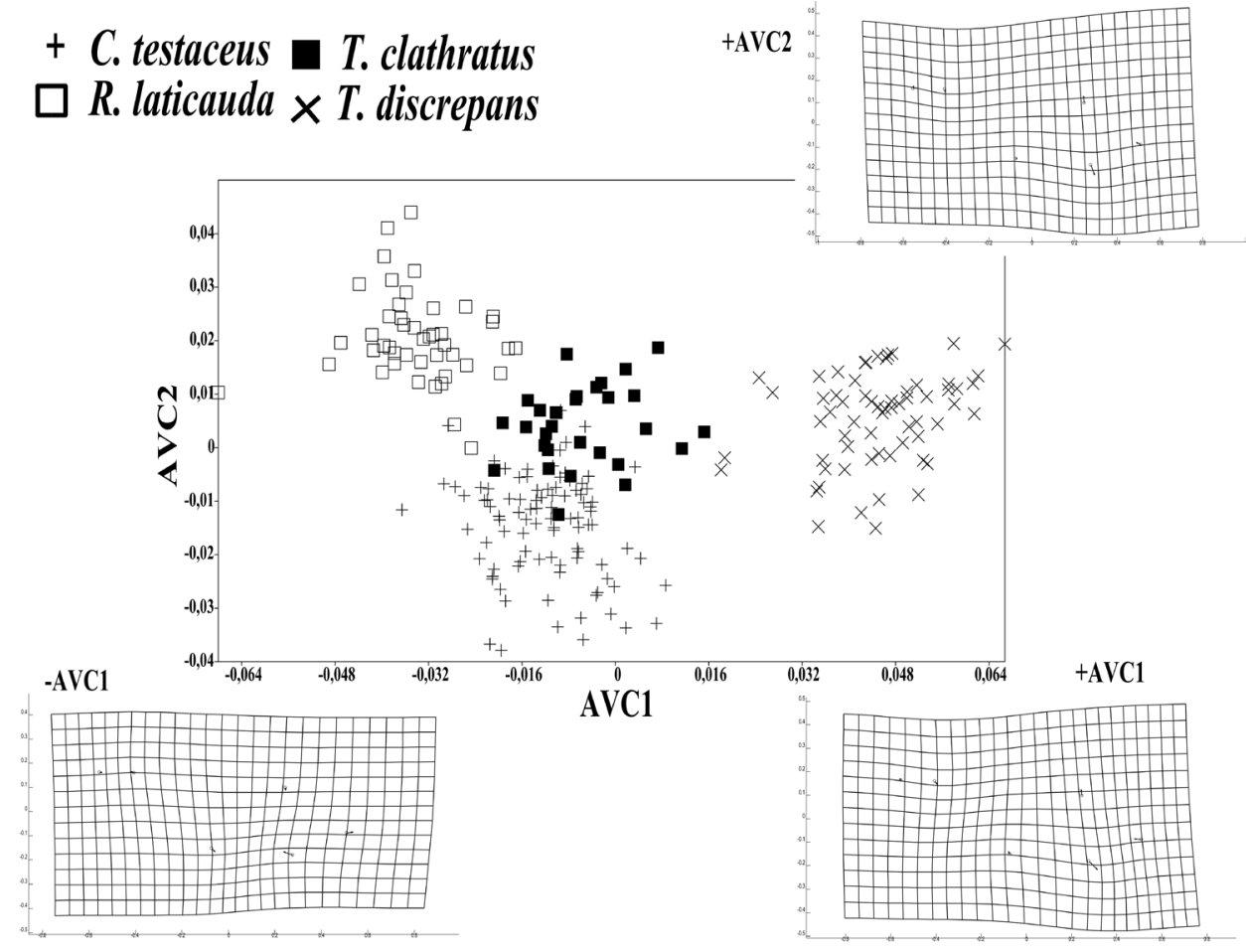

Figura 2. Diagrama de los 2 primeros factores canónicos del AVC para los 233 especímenes de Buthidae y Scorpionidae, mostrando rejillas de deformación en las conformaciones de la patela.

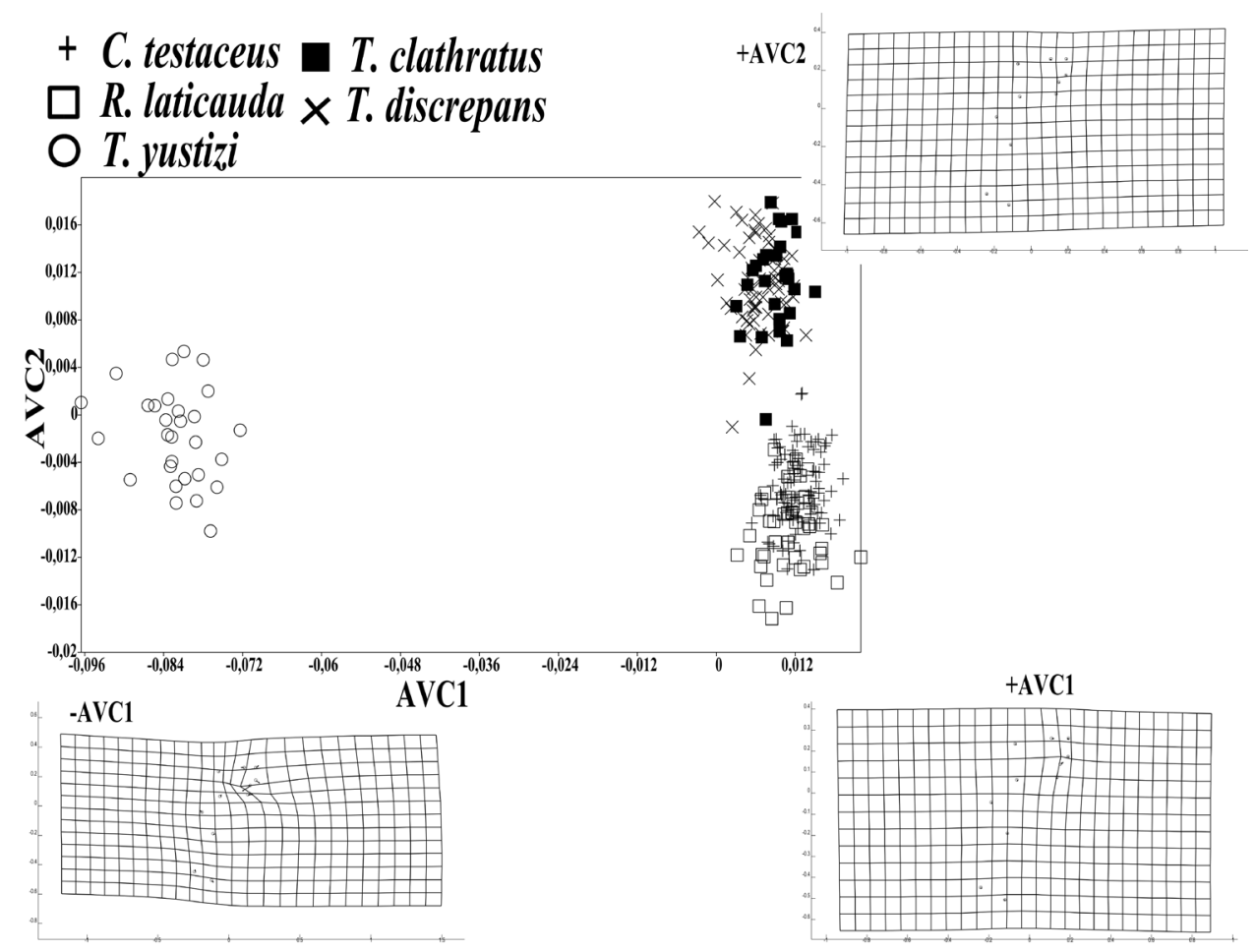

Figura 3. Diagrama de los 2 primeros factores canónicos del AVC para los 264 especímenes de Buthidae y Scorpionidae, mostrando rejillas de deformación en las conformaciones de la coxas. 


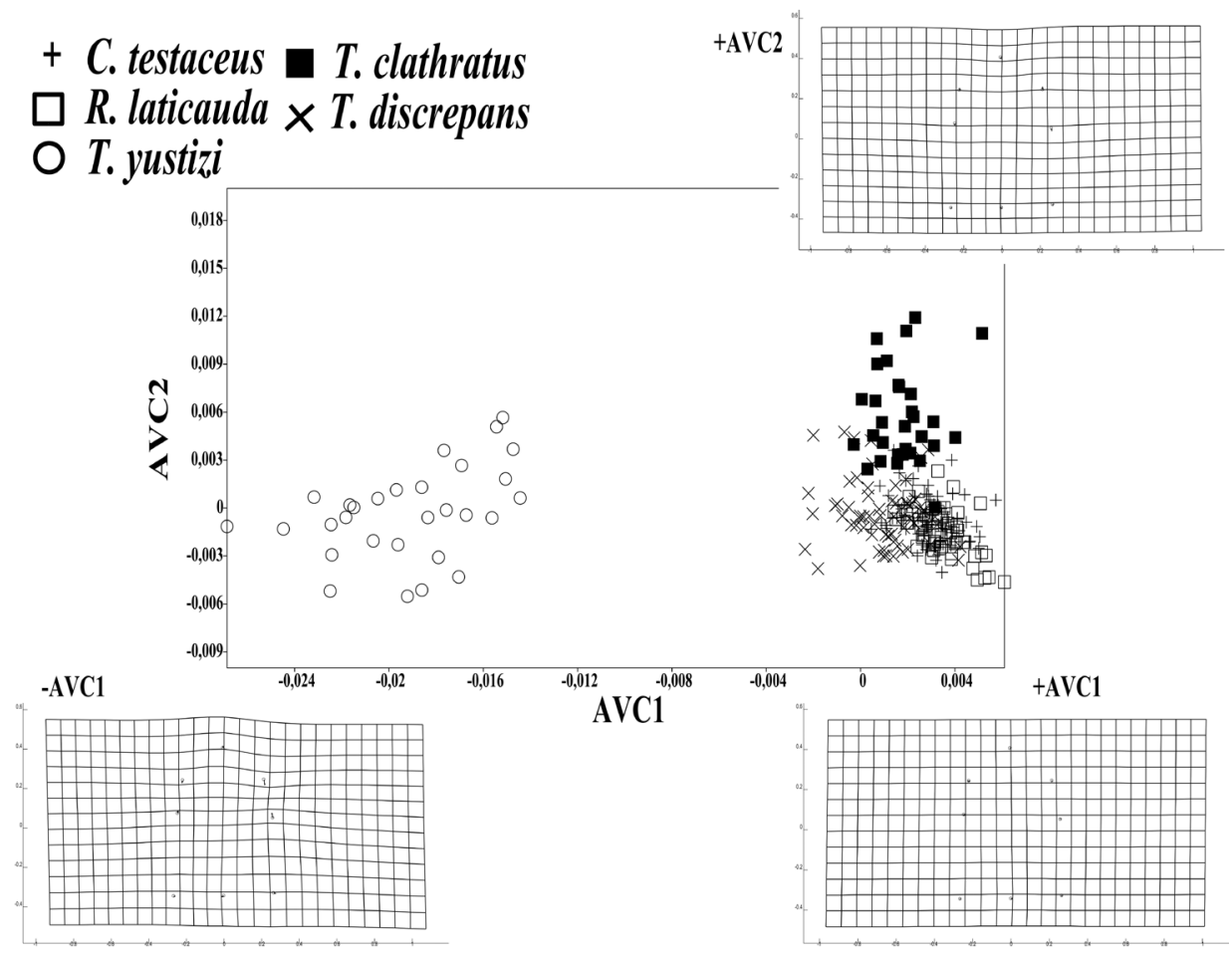

Figura 4. Diagrama de los 2 primeros factores canónicos del AVC para los 264 especímenes de Buthidae y Scorpionidae, mostrando rejillas de deformación en las conformaciones del esternón.

(Fig. 5) muestra la separación de T. yustizi, solapamiento parcial entre $T$. discrepans y $T$. clathratus y solapamiento entre $R$. laticauda y $C$. testaceus. En cuanto a las deformaciones, C. testaceus mostró muy poca variación con respecto a CC. Luego en R. laticauda el PAR 8 se desplaza levemente en dirección al 1. Por otro lado, la configuración de $T$. clathratus varía respecto a la $\mathrm{CC}$, particularmente en los PAR 2 y 4 , los cuales se desplazan hacia 6 y 5 , respectivamente. En seguida los PAR 9 y 10 se desplazan hacia arriba. En $T$. discrepans se aprecia la mayor deformación, donde los PAR 2 y 4 se mueven hacia arriba, alejándose de 1 y 5 . Mientras que los PAR 6 y 7 se acercan al 9 , y 8 se aleja de 7 y 9 y a su vez el 10 y 9 se mueven hacia 2 y 6 , respectivamente. Por último, en T. yustizi se observa que los PAR 2, 3 y 4 se alejan de 10,9 y 8 , respectivamente y los PAR 13,12 y 11 se acercan a 5,6 y 7 .

Quela del pedipalpo. Se encontraron diferencias significativas $(\mathrm{P}<0.05)$ entre el tamaño isométrico de todas las especies con excepción de T. yustizi y T. clathratus, siendo T. clathratus $(8.06 \mathrm{~mm})$ la de menor tamaño, seguida de $T$. yustizi $(9.29 \mathrm{~mm})$, C. testaceus $(10.04 \mathrm{~mm})$, luego R. laticauda $(10.75 \mathrm{~mm})$ y por último $T$. discrepans $(13.55 \mathrm{~mm})$. Se presentan los resultados de la prueba de reclasificación en cada eje discriminante del AVC: $\Lambda^{1}=0.0035, \chi^{2} 1419.01$, $80 \mathrm{gl} p<0.0001, \Lambda^{2}=0.1099, \chi^{2} 557.98,57 \mathrm{gl} p<0.0001$,
$\Lambda^{3}=0.4187, \chi 2218.95,36$ gl y $p<0.0001$ y $\Lambda^{4}=0.6851, \chi 2$ $95.10,17 \mathrm{gl} \mathrm{y} p<0.0001$. La clasificación de 266 individuos fue perfecta al $100 \%$ en T. yustizi (30/30), seguidos de $96 \%$ T. clathratus (29/31) y T. discrepans $(62 / 64), 94 \%$ C. testaceus (91/96) y $82 \%$ en $R$. laticauda (37/45). El diagrama factorial del AVC (Fig. 6) muestra la separación de T. yustizi, solapamiento parcial de T. clathratus y $T$. discrepans y solapamiento entre $C$. testaceus y $R$. laticauda. En C. testaceus el pedipalpo se comprime entre los PAR 10,9 y 8 respecto a 4, 5 y 6. Mientras en $R$. laticauda los PAR 9 y 8 se alejan de 5 y 6 , respectivamente. Por otra parte, en $T$. clathratus se observa aproximación de los PAR 9 y 8 al 5 y 6 , respectivamente. Seguidamente en $T$. discrepans los PAR 10, 9 y 8 se aproximan a 4, 5 y 6, respectivamente. Por último, T. yustizi, muestra una marcada variación en su configuración: el PAR 1 que se acerca al 2, y éste, a su vez, se desplaza en dirección diagonal inferior, y los PAR 11, 10, 9 y 8 se alejan de manera horizontal de $3,4,5$, y 6 , respectivamente.

\section{Discusión}

Tamaño de patela, coxas, esternón, caparazón y quela. La variación de talla de la patela en las especies estudiadas es congruente con lo reportado en la literatura para las dimensiones totales; T. clathratus está caracte- 


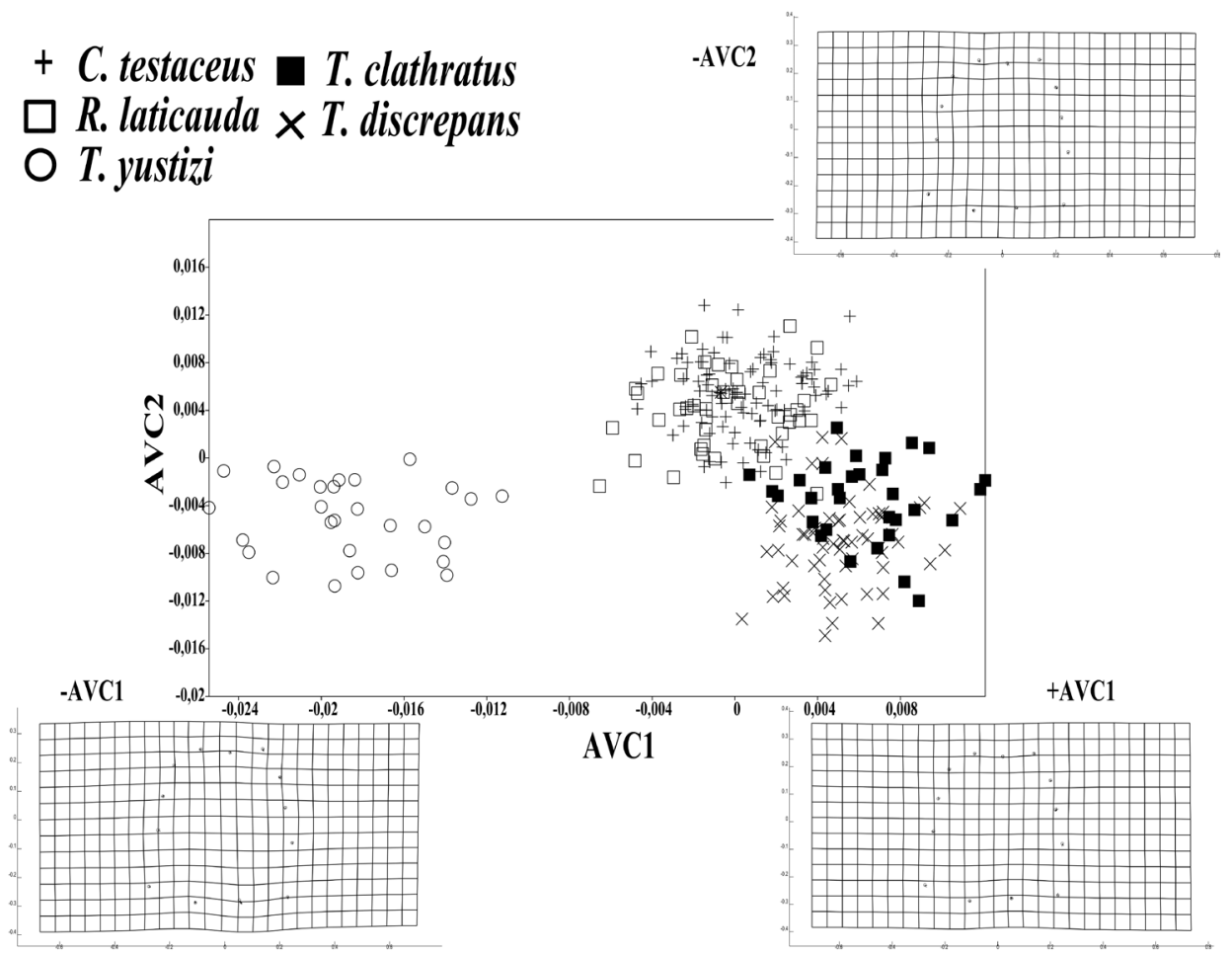

Figura 5. Diagrama de los 2 primeros factores canónicos del AVC para los 264 especímenes de Buthidae y Scorpionidae, mostrando rejillas de deformación en las conformaciones del caparazón.

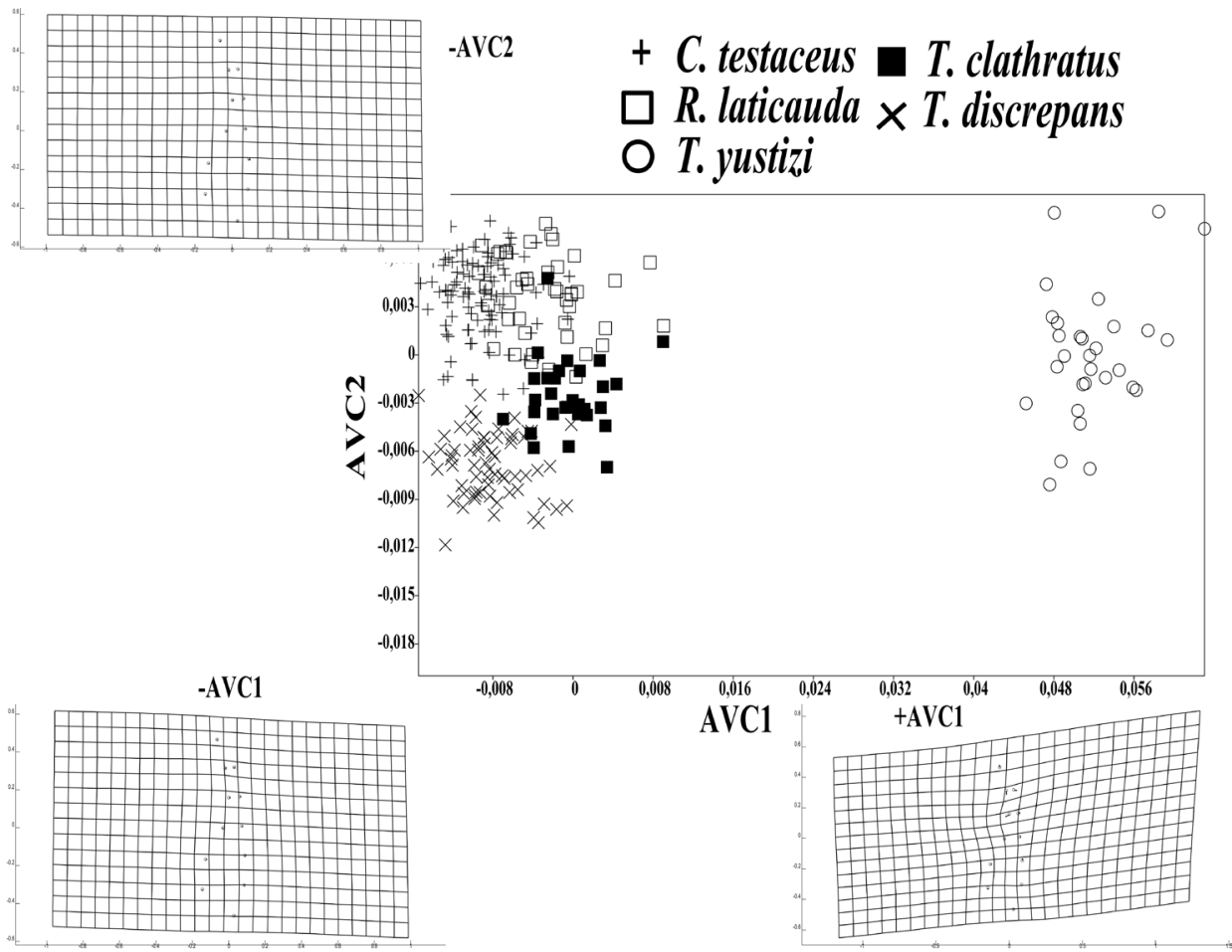

Figura 6. Diagrama de los 2 primeros factores canónicos del AVC para los 266 ejemplares de Buthidae y Scorpionidae, mostrando rejillas de deformación en las conformaciones de la quela. 
rizada por su tamaño reducido (González-Sponga, 1996; Rojas-Runjaic y De Armas, 2007) en comparación con $R$. laticauda y $C$. testaceus que poseen dimensiones similares (González-Sponga, 1996, 2005; Teruel y Tietz, 2008). Seguidamente las coxas de T. yustizi son poco alargadas, lo cual contribuye a la similitud en el tamaño del centroide en $T$. clathratus. Sin embargo, al comparar la talla total de ambas especies, $T$. clathratus posee menor tamaño que T. yustizi (González-Sponga, 1996; de Armas y RojasRunjaic, 2006). En cuanto a las estructuras restantes: esternón, caparazón y quela, las diferencias de tamaño se correspondieron con la talla total de las especies, siendo T. clathratus la de menor talla y $T$. discrepans la de mayor tamaño. Manzanilla-Puppo (1992) estudió poblaciones de $R$. laticauda en Venezuela, empleando para ello el método de cerchas (Truss network) y análisis de componentes principales (ACP) en 4 estructuras, de las cuales fue incluido el caparazón. El ACP y cálculo de coeficientes alométricos muestran que el prosoma representa una estructura apropiada para estudios morfométricos (Sissom et al., 1990). Sin embargo, el análisis de cerchas no permitió la separación de individuos provenientes de diferentes localidades estudiadas, por lo cual sugiere que el patrón de alometrías es complejo y se requiere de estudios más detallados. Vásquez et al. (2001) estudiaron la variación intraespecífica en Diplocentrus sp. de Venezuela, mediante análisis univariados en ancho del prosoma, longitud de meso y metasoma. Particularmente, al comparar estas longitudes y la fórmula de las espinas en los tarsos 3 y 4, con el material tipo de Tarsoporosus yustizi (= Diplocentrus yustizi) sugieren la existencia de una nueva especie. Adicionalmente determinan que el ancho anterior y posterior de prosoma, largo y ancho del segmento $\mathrm{V}$ del metasoma, permiten la diferenciación entre machos y hembras. Ozkan et al. (2006) caracterizaron el dimorfismo sexual en Androctonus crassicauda (Oliver, 1807) mediante diferencias métricas en ancho del caparazón, longitud del peine y número de dientes pectíneos. Santibáñez-López y Ponce-Saavedra (2009) en la descripción de 2 especies mexicanas de Centruroides, realizaron un ACP con base en variables morfométricas, merísticas y discretas, que incluían el largo de caparazón, mesosona, segmentos I-V, dedo móvil de la quela, desarrollo del tuberculo subaculear, entre otras. Particularmente la relación entre longitud del caparazón y largo del segmento $\mathrm{V}$, permitió la diferenciación entre C. serrano Santibáñez-López y Ponce-Saavedra 2009 y C. baergi Hoffmann 1932. Diferencias de conformación. La disposición de las tricobotrias en Scorpiones es importante en diversos estudios tanto taxonómicos como filogenéticos (Vachon, 1973; Soleglad, 1976; GonzálezSponga, 1996; Prendini, 2000; Quiroga et al., 2004; Fet et al., 2005; Soleglad et al., 2005, 2009; Botero-Trujillo, 2008). Fet et al. (2005) analizaron la disposición de las tricobotrias dorsales de la patela con respecto a la carena dorsal mediana (DMc) y puntualmente sobre la tricobotria $d_{3}$, indicando que ésta puede desplazarse hacia la DMc o alejarse. En este trabajo, los resultados muestran un comportamiento diferente, puesto que los PAR fueron colocados sobre las tricobotrias $d_{1}-d_{5}$ y la tricobotria $i$, abordando así sólo la conformación de éstas sin considerar la posición de la Dmc. No obstante, se determinó que la conformación muestra desplazamientos para $T$. discrepans y $R$. laticauda en $d_{4}, d_{5}$ e $i$, a diferencia de $C$. testaceus y T. clathratus, donde las configuraciones no mostraron desplazamientos marcados. Estos resultados indican la existencia de una valiosa información en estas 4 tricobotrias, lo cual sugiere la importancia de considerar estos caracteres en la separación de algunos grupos taxonómicos que presentan problemas en su identificación (De Armas y Rojas-Runjaic, 2006).

En el estudio morfométrico realizado por Soleglad y Fet (2003) sobre el esternón y las coxas, se emplearon relaciones lineales entre las longitudes de las coxas II-IV, así como diferentes medidas del esternón, y relaciones entre esternón y coxas. Luego de revisar más de 100 especies, determinaron 2 formas fundamentales de esternón, las cuales se denominan tipo 1 y tipo 2, caracterizadas por presentar una depresión posterior, la cual no bisecta el borde posterior del esternón y con una invaginación posterior y lóbulos laterales convexos, respectivamente. De acuerdo con estas características, las Buthidae y Scorpionidae presentan esternón tipo 1 y tipo 2, respectivamente. En T. discrepans y $T$. clathratus, existe un ligero ensanchamiento en la región medial del esternón, el cual no se aprecia en $R$. laticauda y C. testaceus; así también, se observa mayor compresión en la región media anterior del esternón. Esto es congruente con la hipótesis de compresión horizontal propuesta por Soleglad y Fet (2003), la cual es más acentuada en estos 2 géneros respecto a las especies de Tityus. En las coxas, se observaron patrones de desplazamiento de los PAR, lo que permite una separación según la variación de la configuración. De esta manera $R$. laticauda y $C$. testaceus, muestran básicamente el mismo acercamiento de los PAR 3 y 6 al PAR 2 y 1 , lo que podría relacionarse con la similitud entre ambos géneros (Lourenço, 1979; Fet et al., 2003, 2005). Soleglad y Fet (2003) sugieren que el aparente alargamiento de la coxas III y IV es evidente en la mayoría de los géneros de Buthidae, y en la mayoría de los géneros de esternón tipo 2 , sugiriendo que este alargamiento es causado, en parte, por la compresión horizontal del esternón, donde las coxas III y IV se extienden hacia dentro para empalmarse al esternón.

La quela de los escorpiones ha sido utilizada en la determinación del sexo y como carácter en las claves de 
identificación (González-Sponga, 1996, 2005; RojasRunjaic, 2004; Teruel y Tietz, 2008). Particularmente $R$. laticauda presenta una quela globosa característica del resto de las Buthidae (González-Sponga, 1996; Teruel y Tietz, 2008). Luego en C. testaceus mostró menor ancho entre los PAR 4 y 10, lo cual se debe a que los dedos de la quela son un tanto más alargados respecto a la mano de la quela. Otro aspecto a considerar, es el número de radios (Fans) seleccionados, para la digitalización de PAR deslizantes; estos probablemente no estén considerando toda la información del contorno de la quela. GonzálezSponga $(1996,2005)$ indica respecto a $T$. clathratus y $T$. discrepans, que la forma de la mano en ambos es globosa a ovoide en machos y estrecha en hembras. Por otro lado, la conformación de T. yustizi es notablemente diferente al resto de las especies, donde se observa una quela más globosa, así como el acortamiento de ésta. La mayoría de las especies pertenecientes a Scorpionidae poseen quelas robustas, en contraparte a las formas estrechas de Buthidae (González-Sponga, 1996). Las diferencias en la forma de la quela en ambas familias, se ha asociado con el grado de toxicidad del veneno. Tradicionalmente se ha indicado que las especies cuyo veneno es altamente tóxico no necesitan, en igual medida, la fuerza proporcionada por quelas robustas. Sin embargo, existen excepciones a esta aseveración, por lo cual aún son necesarios estudios fisiológicos, morfológicos, biomecánicos y evolutivos, a fin de demostrar esta relación. Recientemente, Carrera et al. (2009) realizaron un estudio morfométrico sobre los quelíceros, determinando la correlación entre el ángulo de la curvatura del dedo móvil en machos y hembras en especies que presentaban un comportamiento de "agarre" durante el cortejo; no obstante, los autores obtienen que no pueden generalizarse tales diferencias respecto al comportamiento de agarre.

Análisis morfométricos y su aplicación en escorpiones. Las herramientas morfométricas han demostrado su utilidad en la discriminación de artrópodos, particularmente en insectos vectores que no pueden identificarse por medio de caracteres morfológicos tradicionales, así como en estudios de variabilidad intra-específica (Dujardin et al., 1997, 1998; Rubio-Pallis, 1998; Calle et al., 2002, 2008). Recientemente, Calle et al. (2008), utilizando la información contenida en la conformación del ala, lograron distinguir las hembras de 11 especies del subgénero Nyssorhynchus de Anopheles presentes en Colombia e indican que estos resultados permiten proponer como apoyo en la sistemática de este taxón la utilización de los puntos anatómicos seleccionados. En otro contexto, Soto-Vivas et al. (2011) realizaron el primer estudio filogenético que contempla el uso de caracteres morfométricos en 10 especies de Rhodniini, sugiriendo que la estimación del cambio de forma es más apropiada para la taxonomía de un grupo cuando se le da el enfoque filogenético, para el análisis de las configuraciones de coordenadas después de la superposición Procrustes y el cálculo de las deformaciones relativas.

En conclusión, los resultados presentados en este trabajo utilizando estructuras como patela, quela, coxas, esternón y caparazón, evidencian por primera vez la importancia de esta herramienta en los estudios de taxonomía y sistemática en escorpiones. La morfometría geométrica, al describir los cambios de forma a partir de la separación de sus componentes más importantes (tamaño y configuración), abre innumerables posibilidades para la realización de análisis inter e intraespecíficos, así como la aplicación en áreas como taxonomía, sistemática, biomecánica, etología y ecología de escorpiones.

\section{Agradecimientos}

A Carlos Sevcik, Gina D’Suze y Alejandro Tampoa, por el préstamo de los ejemplares del Laboratorio de Neurofarmacología Celular del Instituto Venezolano de Investigaciones Científicas. A Fernando Rojas, por su colaboración en el suministro del material bibliográfico. A Ana Soto-Vivas y demás miembros del Centro de Estudios en Enfermedades Endémicas y Salud Ambiental, Adscrito al IESP “Dr. Arnoldo Gabaldon”, por facilitar equipos ópticos y espacio de trabajo.

\section{Literatura citada}

Belen, A., B. Alten y A. M. Aytekin. 2004. Altitudinal variation in morphometric and molecular characteristics of Phlebotomus papatasi populations. Medicine and Veterinary Entomology 18:343-350.

Bookstein, F. L. 1982. Foundation of morphometrics. Annual Review of Ecology and Systematics 13:451-470.

Bookstein, F. L. 1996. Landmark methods for forms without landmarks: morphometrics of group differences in outline shape. Medical Image Analysis 1:225-243.

Botero-Trujillo, R. 2008. The scorpion genus Ananteris in Colombia: comments on the taxonomy and description of two new species (Scorpiones, Buthidae). The Journal of Arachnology 36:287-299.

Calle D. A., M. Quiñónez, H. Erazo y N. Jaramillo. 2002. Morphometric discrimination of females of five species of Anopheles of the subgenus Nyssorhynchus from Southern and Northwest Colombia. Memorias do Instituto Oswaldo Cruz 97:1191-1195.

Calle, D. A., M. Quiñónez, H. Erazo y N. Jaramillo. 2008. Discriminación por morfometría geométrica de once especies de Anopheles (Nyssorhynchus) presentes en Colombia. Biomedica 28:371-385.

Carrera, P. C., C. I. Mattoni, y A. V. Peretti. 2009. Chelicerae as male grasping organs in scorpions: sexual dimorphism and 
associated behaviour. Zoology 112:332-350.

De Armas, L. F. y F. J. Rojas-Runjaic. 2006. On the poorly known genus Mesotityus González-Sponga, 1981 (Scorpiones: Buthidae). Euscorpius 47:1-11.

De la Riva, J., F. Le Pont, V. Ali, A. Matias, S. Mollinedo y J. P. Dujardin. 2001. Wing Geometry as a tool for studying the Lutzomyia longipalpis (Diptera: Psychodidae). Memorias Instituto Oswaldo Cruz 96:1089-1094.

De Sousa, L., S. Bonoli, M. Quiroga y P. Parrilla. 1996. Scorpion sting in Montes municipality of the state of Sucre, Venezuela: geographic distribution. Revista de Instituto de Medicina Tropical de São Paulo 38:147-152.

De Sousa, L., P. Parrilla-Alvárez y M. Quiroga. 2000. An epidemiological review of scorpion stings in Venezuela: the northeastern region. Journal of Venomous Animal and Toxins 6:127-156

Dujardin J. P., H. Bermúdez, C. Casini, C. J. Schofield y M. Tibayrenc. 1997. Metric differences between sylvatic and domestic Triatoma infestans (Hemiptera: Reduviidae) in Bolivia. Journal of Medical Entomology 34:544-551.

Dujardin J. P., G. Forgues, M. Torrez, E. Martínez, C. Córdoba y A. Gianella. 1998. Morphometrics of domestic Panstrongylus rufotuberculatus in Bolivia. Annals of Tropical Medicine and Parasitology 92:219-228.

Dvorak, V., A. M. Aytekin, B. Alten, S. Skarupova, J. Votypka y P. Volf. 2006. A comparison of the intraspecific variability of Phlebotomus sergenti Parrot, 1917 (Diptera: Psychodidae). Journal of Vector Ecology 31:229-238.

Fet, V., M. E. Soleglad y G. Lowe. 2005. A new trichobothrial character for the high-level systematics of Buthoidea (Scorpiones: Buthida). Euscorpius 23:1-40

Fet, V., B. Gantenbein, A. V. Gromov, G. Lowe y W. R. Lourenço. 2003. The first molecuar phylogeny of Buthidae (Scorpiones). Euscorpius 4:1-10.

Fet, V., W. D. Sissom, G. Lowe y M. E. Braunwalder. 2000. Catalog of the scorpions of the world (1758-1998). The New York Entomological Society. 690 p.

González-Sponga, M. A. 2005. Arácnidos de Venezuela. Redescripción de Tityus discrepans (Karsch, 1879) (Scorpionida: Buthidae). Memoria de la Fundación La Salle de Ciencias Naturales 64:91-100.

González-Sponga, M. A. 1996. Guía para identificar escorpiones de Venezuela. Cuadernos Lagoven. 204 p.

Guinand, A., H. Cortés, G. D'suze, P. Díaz, C. Sevcik, M. GonzálezSponga y G. Eduarte. 2004. Escorpionismo del género Tityus en la sierra falconiana y su correlación con la liberación de mediadores inflamatorios y enzimas cardíacas. Gaceta Médica de Caracas 112:131-138.

Jaramillo, N. D. y C. M. Wolff. 2002. Geometric morphometric differences between Panstrongylus geniculatus from field and laboratory. Memorias do Instituto Oswaldo Cruz 97:667673.

Lourenço, W. R. 1979. A propôs de la véritable identité des genres Rhopalurus Thorell, 1876 et Centruroides Marx, 1889 (Scorpiones, Buthidae). Revue Arachnologique 2:213-219.

Lourenço, W. R. 1994. Biogeographic patterns of tropical South American scorpions. Studies on Neotropical Fauna and Environment 29:219-231.
Lourenço, W. R. 2000. Panbiogeographie, les familles des scorpions et leur repartition geographique. Biogeographica 76:21-39.

Lourenço, W. R. 2002. Scorpions of Brazil. Les editions de l'If, Paris. 307 p.

Lourenço, W. R. y R. P. Otero. 1998. Tityus antioquensis sp.n. a new species of scorpion from the Departament Antioquia, Central Cordillera of Colombia (Scorpiones, Buthidae), with a check list and key for the Colombian species of the genus. Entomologische Mitteilungen aus dem Zoologischen Museum Hamburg 12:297-307.

Machado, A. y A. Rodríguez-Acosta. 1997. Los animales venenosos y ponzoñosos de Venezuela. Consejo de Desarrollo Científico y Humanístico, Universidad Central de Venezuela, Caracas. $11 \mathrm{p}$.

Manzanilla-Puppo, J. 1992. Género Rhopalurus Thorell (Scorpionida: Buthidae) en Venezuela. Consideraciones biogeográficas taxonómicas y ecológicas. Tesis, Maestría Instituto de Zoología Agrícola, Facultad de Agronomía, Universidad Central de Venezuela, Maracay, Aragua. 171 p.

Matías, A. J. X., M. De La Riva y J. P. Dujardin. 2001. Rhodnius robustus in Bolivia Identified by its Wings. Memorias do Instituto Oswaldo Cruz 96:947-950.

Ozkan, O., Z. Adiguzel y S. Kar. 2006. Parametric values of Androctonus crassicauda (Oliver, 1807) (Scorpiones: Buthidae) from Turkey. Journal of Venomous Animal and Toxins including Tropical Disease 12:549-559.

Polis, G. A. 1990. Ecology. In The biology of scorpions, G. A. Polis (ed.). Stanford University Press, California. p. 247-293.

Prendini, L. y W. C. Wheeler. 2005. Scorpion higher phylogeny and classification, taxonomic anarchy, and standards for peer review in online publishing. Cladistics 21:446-494.

Prendini, L. 2000. Phylogeny and classification of the superfamily Scorpionoidea Latreille 1802 (Chelicerata, Scorpiones): an exemplar approach. Cladistics 16:1-78.

Prendini, L. 2001. Substratum specialization and speciation in southern African scorpions: the effect hypothesis revisited. In Scorpions. In memoriam Gary A. Polis, V. Fet y P. A. Selden (eds.). British Arachnological Society, Burnham Beeches, Bucks. p.113-138.

Prendini, L. 2003a. Discovery of the male of Parabuthus mulleri, and implications for the phylogeny of Parabuthus (Scorpiones: Buthidae). American Museum Novitates 3408:1-24.

Prendini, L. 2003b. A new genus and species of bothriurid scorpion from the Brandberg Massif, Namibia, with a reanalysis of bothriurid phylogeny and a discussion of the phylogenetic position of Lisposoma Lawrence. Systematic Entomology 28:149-172.

Prendini, L. 2003c. Systematics and biogeography of the family Scorpionidae (Chelicerata: Scorpiones), with a discussion on phylogenetic methods. Invertebrate Systematics 17:185-259.

Quiroga, M. L. De Sousa, P. Parrilla-Álvarez y J. Manzanilla. 2004. The first report of Tityus (Scorpiones: Buthidae) in Anzoátegui State, Venezuela. A new species. Journal of Venomous Animal and Toxins including Tropical Disease 1:10-33.

Reyes-Lugo, M. y A. Rodríguez-Acosta. 2001. Scorpion envenoming by Tityus discrepans Pocock, 1897 in the northern coastal region of Venezuela. Revista Científica FCV/LUZ 
$11: 412-417$

Rohlf, F. J. 2003. tpsRelw, Program provides a low dimensional approximation (via a principal components analysis) to the tangent space approximation of shape space, ver. 1.37. Department of Ecology and Evolution, State University of New York, Stony Brook. http://life.bio.sunysb.edu/morph/ index.html;última consulta: 05.VIII.2011.

Rohlf, F. J. 2007. tpsDig, Program for digitalizing morphologic landmark and outlines for geometric morphometric analyses, ver. 2.04. Department of Ecology and Evolution, State University of New York, Stony Brook. http://life.bio.sunysb. edu/morph/index.html;última consulta: 05.VIII.2011.

Rojas-Runjaic, F. J. 2004. Un nuevo escorpión del género Chactas Gervais, 1844 (Scorpiones: Chactidae) de una cueva de la sierra de Perijá, Venezuela. Revista Ibérica de Aracnología 10:245-253.

Rojas-Runjaic, F. J. y L. F De Armas. 2007. Dos nuevas especies venezolanas del grupo Tityus clathratus y notas sobre Tityus ramirezi Esquivel de Verde, 1968 (Scorpiones: Buthidae). Boletín de la Sociedad Entomológica Aragonesa 41:53-66.

Rojas-Runjaic, F. J. y L. De Sousa. 2007. Catálogo de los escorpiones de Venezuela (Arachnida: Scorpiones). Boletín de la Sociedad Entomológica Aragonesa 40:281-307.

Rojas-Runjaic, F. J. y A. C. Becerra. 2008. Diversidad y distribución geográfica de la escorpiofauna del estado Zulia, Venezuela. Boletín del Centro de Investigaciones Biológicas, Universidad del Zulia 42:461-477.

Rubio-Pallis, Y. 1998. Caracterización morfométrica de poblaciones de Anopheles (Nyssorhynchus) darlingi del sur de Venezuela. Boletín Entomología Venezolana 13:141-172.

Santibánez-López, C. y J. Ponce-Saavedra. 2009. A new species of Centruroides (Scorpiones: Buthidae) from the northern mountain range of Oaxaca, Mexico. Revista Mexicana de Biodiversidad 80:321-331

Sheets, H. D. 2005a. CoordGen6, coordinate generation program for calculating shape coordinate. www3.canisius.edu/ sheets/ morphsoft.html;última consulta: 05.VIII.2011.

Sheets, H. D. 2005b. CVAGen6, canonical variates analysis program for the analysis of shape, based on partial warp scores. www3.canisius.edu/ sheets/morphsoft.html;última consulta: 05.VIII.2011.

Sheets, H. D. 2005c. MakeFan, a tool for drawing alignment "fans" at equal angular spacing. www3.canisius.edu/ sheets/ morphsoft.html;última consulta: 05.VIII.2011.

Sissom, D. W. 1990. Systematics, biogeography and paleontology. In The biology of scorpions, G. Polis (ed.). Stanford University
Press, California. p. 64-160.

Soleglad, M. E. 1976. The taxonomy of the genus Hadrurus based on chela trichobothria (Scorpionida: Vejovidae). The Journal of Arachnology 3:113-134.

Soleglad, M. E. y V. Fet. 2003. The scorpion sternum: structure and phylogeny (Scorpiones: Orthosterni). Euscorpius 5:1-33.

Soleglad, M. E., V. Fet y F. Kovařik. 2005. The systematic position of scorpion genera Heteroscorpion Birula, 1903 and Urodacus Peters, 1861 (Scorpiones: Scorpionoidea). Euscorpius 20:138.

Soleglad, M. E., F. Kovařik y V. Fet. 2009. Etudes on iurids, I. The orthobothriotaxic pattern of Iuridae, with observations on neobothriotaxy in genus Iurus (Scorpiones: Iuroidea). Euscorpius 79:1-23.

Soto-Vivas, A., J. Liria y E. de Luna. 2011 Morfometría geométrica y filogenia en Rhodniini (Hemiptera, Reduviidae) de Venezuela. Acta Zoologica Mexicana 27:87-102.

Stockwell, S. A. 1992. Systematics observations on North American Scorpionida with a checklist of the family and genera. Journal of Medical Entomology 29:407-422.

Teruel, R. y A. K. Tietz. 2008. The true identity of Rhopalurus pintoi Mello-Leitão, 1932, with notes on the status and distribution of Rhopalurus crassicauda Caporiacco, 1947 (Scorpiones: Buthidae). Euscorpius 70:1-16.

Vachon, M. 1973. Éstude des caracteres utilisés pour classer les familles et les genres de Scorpions (Arachnides). Bulletin du Muséum National D'histoire Naturelle 140:857-958.

Van der-Molen, S., N. A. Martínez y J. R. González. 2007. Introducción a la morfometría geométrica: curso teóricopráctico. Universitat de Barcelona.82 p.

Vasquez, C., E. Yustiz, I. Morales, J. Gallardo y Y. Rios. 2001. Variación intraespecífica en una población de Diplocentrus sp. Agronomía Tropical 51:563-574.

Villegas, J., M. D. Feliciangeli y J. P. Dujardin. 2002. Wing shape divergence between Rhodnius prolixus from Cojedes (Venezuela) and Rhodnius robustus from Mérida (Venezuela). Infection, Genetics and Evolution 2:121-128.

Volschenk, S. E. 2005. A new technique for examining surface morphosculpture of scorpions. The Journal of Arachnology 33:820-825.

Warburg, M. R. y G. A. Polis. 1990. Behavioral responses, rhythms and activity patterns. In The biology of Scorpions, G. A. Polis (ed.). Stanford University Press, California. p. 224-246.

Zelditch, M., D. L. Swiderski, H. D. Sheets y W. L Fink. 2004. Geometric morphometrics for biologists: a primer. Elsevier Academic, Boston, Massachusetts. 443 p. 